\title{
Compaction through Buckling in 2D Periodic, Soft and Porous Structures: Effect of Pore Shape
}

\section{Citation}

Overvelde, Johannes Tesse Bastiaan, Sicong Shan, and Katia Bertoldi. 2012. Compaction through buckling in 2D periodic, soft and porous structures: Effect of pore shape. Advanced Materials 24(17): 2337-2342.

\section{Published Version}

doi:10.1002/adma.201104395

\section{Permanent link}

http://nrs.harvard.edu/urn-3:HUL.InstRepos:10919788

\section{Terms of Use}

This article was downloaded from Harvard University's DASH repository, and is made available under the terms and conditions applicable to Open Access Policy Articles, as set forth at http:// nrs.harvard.edu/urn-3:HUL.InstRepos:dash.current.terms-of-use\#OAP

\section{Share Your Story}

The Harvard community has made this article openly available.

Please share how this access benefits you. Submit a story.

\section{Accessibility}




\title{
Compaction through buckling in 2D periodic, soft and porous structures: effect of pore shape
}

\author{
J. T. B. Overvelde * ${ }^{\dagger}$, S. Shan ${ }^{* \dagger}$, K. Bertoldi * . \\ *School of Engineering and Applied Sciences, Harvard University, Cambridge, Massachusetts 02138, USA, and †These authors contributed equally.
}

\begin{abstract}
Adaptive structures allowing dramatic shape changes offer unique opportunities for the design of responsive and reconfigurable devices. Artificial morphing and foldable structures have traditionally been fabricated using stiff structural members connected by mechanical joints $[1,2,3,4,5,6]$, resulting in a very challenging manufacturing process at small length scales. In contrast, there are many examples of soft adaptive structures in nature: mimosa leaves fold when touched [7, 8]; venus flytraps open and close to catch prey [10]; mechanical expansion and contraction of the pigment-filled sacks in squids result in dynamic color changes [11]. Applying these natural design principles in new ways could result in a novel class of responsive and reconfigurable devices. Soft structures where the folding mechanisms is induced by a mechanical instability may lead to origami materials that can be easily manufactured over a wide range of length scales. Possible and exciting applications include active materials for on-demand drug delivery, colorful and reconfigurable displays and robots that can squeeze themselves through small openings and into tight spaces.
\end{abstract}

Soft structures with deliberately designed patterns may significantly change their architecture in response to diverse stimuli $[12,13]$, opening avenues for reconfigurable devices that change their shape to respond to or alter their environment. Two-dimensional periodic porous structures recently attracted considerable attention because of dramatic transformations of the original geometry observed as the results of mechanical instabilities $[14,15,16]$. Upon reaching a critical applied stress, a square array of circular holes in an elastomeric matrix is found to suddenly transform into a periodic pattern of alternating, mutually orthogonal ellipses (see Fig. 2A). This behavior has been demonstrated to provide opportunities for the design of materials with tunable negative Poisson's ratio [17], phonic switches [18] and strain-tunable optomechanical materials [19]. However, so far only the response of structures with circular and elliptical holes has been investigated and the effect of the pores shape on the structural response has not been explored yet.

Shape plays an important role in the design and performance of materials and engineering devices. Computer analysis - known as shape and topology optimization [20,21,22] - is performed routinely in an effort to identify optimal shapes to improve a certain performance under some constraints. In this study we will investigate both numerically and experimentally the effect of pore shape on the nonlinear response of a square array of holes in a soft matrix. The hole shape is found to provide a convenient parameter to control attractive features of soft porous systems, such as their compaction (quantified as change of structure planar area divided by original area) and negative Poisson's ratio ${ }^{1}$. Our results show that the pore shape can be used effectively to design material with desired properties and pave the way for the development of a new class of soft, active and reconfigurable devices over a wide range of length scales.

Here, we focus on holes with four-fold symmetry and make use of Fourier series expansion to describe their contour as

$$
\begin{gathered}
x_{1}=r(\theta) \cos \theta, \quad x_{2}=r(\theta) \sin \theta, \\
\text { with } r(\theta)=r_{0}\left[1+c_{1} \cos (4 \theta)+c_{2} \cos (8 \theta)\right],
\end{gathered}
$$

where $0 \leq \theta \leq 2 \pi$ and three parameters have been introduced to control the pore size $\left(r_{0}\right)$ and shape $\left(c_{1}\right.$ and $\left.c_{2}\right)$. While $c_{1}=c_{2}=0$ in eqn. 1 provides a description of a circle of radius $r_{0}$, varying $c_{1}$ and $c_{2}$ a variety of shapes can be obtained, as shown in Fig. 1. Thus, in terms of optimization, $c_{1}$ and $c_{2}$ represent a $2 \mathrm{D}$ design space which allows for a systematic study of the effect of shape on the compaction of the structures.
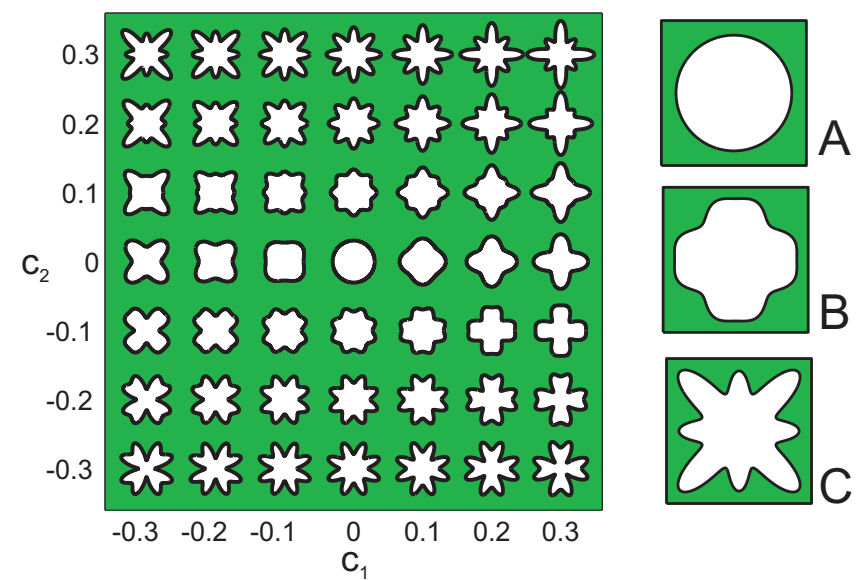

Fig. 1. Left: Shapes obtained using Eqn. 1 and $c_{1}=c_{2}=-0.3: 0.1: 0.3$, while keeping $r_{0}$ constant. Right: Representative volume elements (RVEs) for the three shapes considered in this work, defined by $\left(\phi^{A}, c_{1}^{A}, c_{2}^{A}\right)=$ $(0.46,0,0),\left(\phi^{B}, c_{1}^{B}, c_{2}^{B}\right)=(0.47,0.11,-0.05)$ and $\left(\phi^{C}, c_{1}^{C}, c_{2}^{C}\right)=$ $(0.44,-0.21,0.28)$

To clearly identify the effect of shape on the material response, we focus on a specific arrangement of the pores and consider holes arranged on a square array, so that $r_{0}$ is related to the porosity of the structure $\phi$ through

$$
r_{0}=\frac{L_{0} \sqrt{2 \phi}}{\sqrt{\pi\left(2+c_{1}^{2}+c_{2}^{2}\right)}},
$$

with $L_{0}$ denoting the center-to-center distance between neighboring holes in the undeformed configuration. Note that, to preserve the structural integrity, $c_{1}$ and $c_{2}$ have to be chosen such that $0 \leq x_{1} \leq$ $L_{0} / 2$ and $0 \leq x_{2} \leq L_{0} / 2$.

While the results of an extensive numerical study on the effect of $c_{1}$ and $c_{2}$ on the non-linear material response will be reported elsewhere, here we will focus on two shapes showing qualitative and quantitative remarkably different behaviors, highlighting the important role played by the pore shape. The response of a soft structure

${ }^{1}$ Although the Poisson's ratio is rigorously defined in the framework of linear elasticity, here we extend the concept to finite elasticity and use it to quantify the lateral contraction/expansion of the material. 
with circular holes defined by $\left(\phi^{A}, c_{1}^{A}, c_{2}^{A}\right)=(0.46,0,0)$ is compared to that of structures with pores defined by $\left(\phi^{B}, c_{1}^{B}, c_{2}^{B}\right)=$ $(0.47,0.11,-0.05)$ and $\left(\phi^{C}, c_{1}^{C}, c_{2}^{C}\right)=(0.44,-0.21,0.28)$ (see Fig. 1). Note that the slight variation in porosity between the three structures is related to limited accuracy during the fabrication process.

(a)

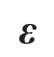

A

B

\section{ㅇ․ㅇ}

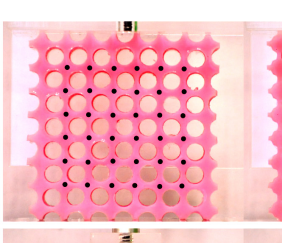

(b)
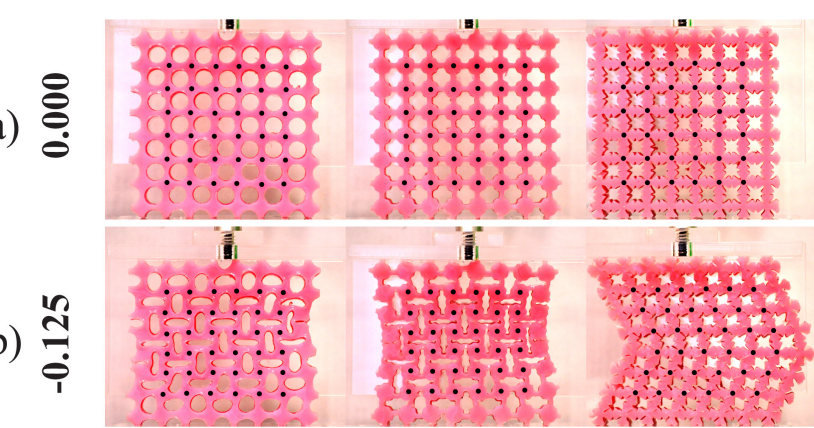

i

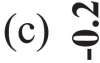

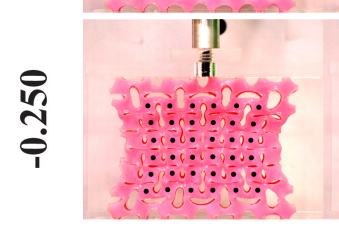

(d)
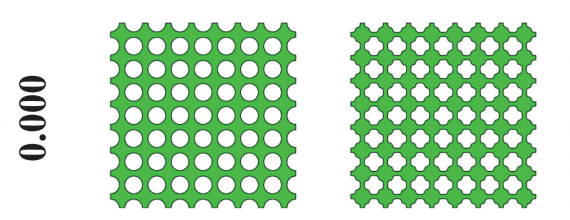

(e)
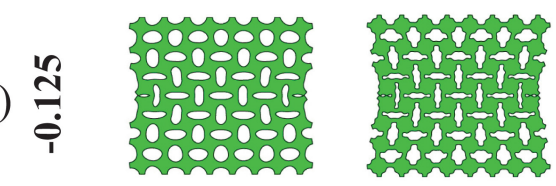

(f)
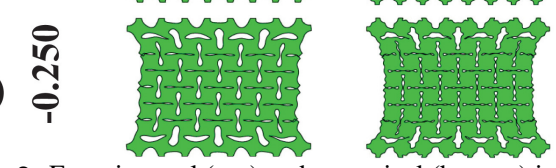

Fig. 2. Experimental (top) and numerical (bottom) images of structures A, B and $\mathrm{C}$ at different levels of applied engineering strain $\epsilon=0,-0.125,-0.25$.

Physical and numerical models of elastomeric structures consisting of $8 \times 8$ unit cells (or representative volume elements, RVEs) arranged on a square array were built as shown in Figs. 2(a) and (d). All the three structures were uniaxially compressed in vertical direction ensuring quasi-static conditions and representative pictures taken during the tests at different level of applied engineering strain $\epsilon$ (calculated as change of structure height divided by the original height) are presented in Fig. 2, showing excellent agreement between experiments and simulations. First, we observe that when a critical value of the compressive strain is reached, buckling occurs, leading to rapid and dramatic changes of the material microstructure. As can be clearly seen in Figs. 2(b) and (e) at a strain $\epsilon=-0.125$ all three structures have buckled. Remarkably, the shape of the holes is found to strongly affect the instability. In structures A and B the critical instability is characterized by a wavelength equal to $2 L_{0}$ in both vertical and horizontal direction and leads to the formation of a checkerboard pattern (see Figs. 2(b) and (e)). By contrast, a buckling mode with a wavelength equal to the size of the sample is observed in structure $\mathrm{C}$, reminiscent of the twinning observed in austenite to martensite phase transformations in shape memory alloys [23]. Once formed, the new pattern becomes further accentuated for increased values of the applied strain, as can be seen in Fig. 2(c) and (f). Remarkably after unloading, the initial shape of the holes was always fully recovered regardless of the amount of applied deformation. Moreover, close inspection of Fig. 2 reveals that the hole shape not only affects the buckling of the structures, but also attractive features such as their lateral contraction and compaction. In structures A and B complete closure of the pores and a significant lateral contraction is observed at $\epsilon=-0.25$, leading to a folded state characterized by an area reduction of $\sim 40 \%$. By contrast, a significantly lower area change at $\epsilon=-0.25$ is found in structure C, highlighting the important role played by microscopic instabilities (i.e. instabilities with wavelengths that are of the order of the size of the microstructure) in the design of novel, soft and foldable systems. Finally, comparison of the pattern in structures A and B at $\epsilon=-0.25$ clearly shows that circular holes do not lead to optimal compaction; an optimized folded configuration that minimized the openings is found in structure $\mathrm{B}$, demonstrating the significant effect that the pore shape has in the design of foldable soft structures.

We used rapid prototyping techniques to fabricate the samples made out of a soft silicone-based rubber. The structures comprise a square array of $8 \times 8$ holes and to reduce the boundaries effect the RVEs closest to all boundaries are cut in half. All structures are characterized by a center-to-center distance between neighboring holes $L_{0}=10 \mathrm{~mm}$, while their out-of-plane thickness is approximately $15 \mathrm{~mm}$. Vinylpolysiloxane was used to fabricate the structures; uniaxial tension test performed on the bulk material revealed that its response up to a stretch of 2 is well captured using an incompressible Neo-Hookean model, with Young's modulus $E^{*}=190 \mathrm{kPa}$. The structures were then compressed at constant speed of $20 \mathrm{~mm} / \mathrm{min}$, while a video was captured and recorded by a high-resolution digital camera facing the specimen. During the tests out-of-plane buckling was prevented holding the specimens with a back plate (see Materials and Methods for more details).

To fully understand the effect of pore shape on the material response, numerical simulations were performed using the non linear finite element code ABAQUS/Explicit. Triangular, quadratic plane strain elements (ABAQUS element type CPE6M) were used and the accuracy of the mesh was ascertained through a mesh refinement study, resulting in a relative mesh density of around 800 to 1250 elements per RVE. An imperfection in the form of the first buckling mode (determined by a linear buckling analysis) was introduced in order to exclude the possibility of the simulation to follow an unstable deformation-path. Quasi-static conditions were ensured by monitoring the kinetic energy and introducing a small damping factor. In the simulations clamped conditions between the specimen and the horizontal fixtures were assumed to simplify the non-adhesive frictional boundary conditions occurring in the experiment.

A more quantitative comparison between the response of the structures investigated in this paper can be made by inspecting the evolution of stress, negative Poisson's ratio and compaction monitored during both experiments and simulations. Fig. 3(a) presents the evolution of the nominal stress $S$ (calculated by dividing the total applied force by the initial cross-sectional area) as function of the applied engineering strain $\epsilon$. For all three structures we observe a behavior typical for cellular solids with three distinct regimes [24]: a linear elastic regime, a stress plateau following thereafter, and densification by further compression. The departure from linearity is a result of buckling and corresponds to a sudden transformation in the periodic pattern as shown in the snapshots of deformed configurations at different levels of strain (Fig. 2). Eventually, at high strains, the holes collapse sufficiently for their boundary to touch, giving rise to the final steep portion of the stress-strain curve. Although all our structures are characterized by a very similar initial 
elastic response, their departure from linearity is well separated and it is found to strongly depend on the pore shape.
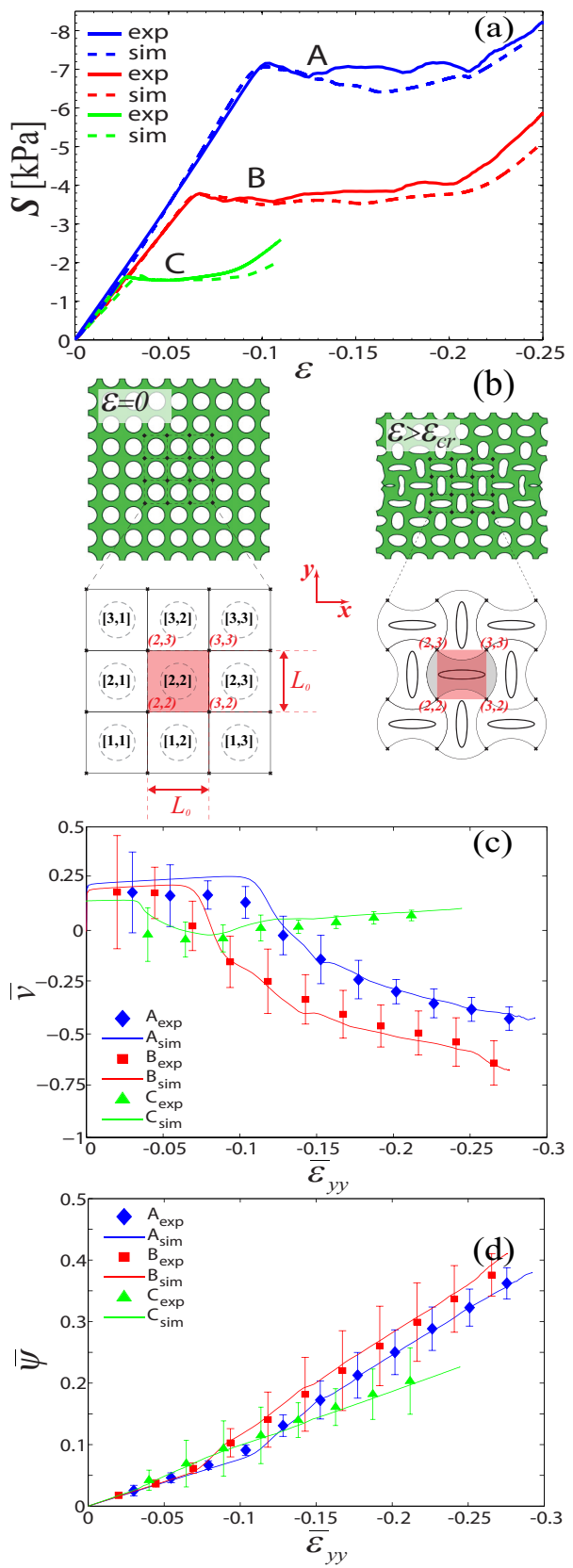

Fig. 3. (a) Experimental and numerical stress-strain curves for the three structures. Solid curves correspond to experiments and dash lines to simulations. (b) Schematic diagram of the central region with 9 RVEs. (c) Evolution of Poisson's ratio $\bar{\nu}$ as a function of local compressive strain $\bar{\epsilon}_{y y}$ for the three structures. Markers correspond to experiments and continuous lines to simulations. (d) Evolution of compaction $\bar{\psi}$ as a function of $\bar{\epsilon}_{y y}$ for the three structures.

It is surprising to observe that the least porous structure (i.e. structure C) is characterized by the lowest buckling stress. This observation clearly confirms that the buckling observed in structures A and $B$ is different in nature from that of structure $C$ and that the shape of the holes has a strong effect on mechanical instability. While structures A and B are characterized by microscopic instabilities, a macro- scopic instability (i.e. instability with a much larger wavelength than the size of the microstructure) is observed in structure C.

To monitor the evolution of the microstructure, we focused on the behavior of the central part of the sample (nine RVEs) where the response was clearly more uniform and not affected by the boundary conditions. The vertices of the nine central RVEs were marked with black dots (see Fig. 3(b)) and their position was recorded using a high-resolution digital camera and then analyzed by digital image processing (Matlab). For each RVE, local values of the engineering strain, $\epsilon_{x x}^{[i, j]}$ and $\epsilon_{y y}^{[i, j]}$, were calculated from the positions of the markers, $x^{(i, j)}$ and $y^{(i, j)}$, as

$$
\begin{aligned}
\epsilon_{x x}^{[i, j]} & =\frac{x^{(i+1, j)}-x^{(i, j)}+x^{(i+1, j+1)}-x^{(i, j+1)}-2 L_{0}}{2 L_{0}} \\
\epsilon_{y y}^{[i, j]} & =\frac{y^{(i+1, j+1)}-y^{(i+1, j)}+y^{(i, j+1)-y^{(i, j)}}-2 L_{0}}{2 L_{0}},
\end{aligned}
$$

where $i, j=1,2,3$ and $L_{0}=10 \mathrm{~mm}$ denotes the distance between markers in the undeformed configuration. The local values of the engineering strain were used to calculate local values of the Poisson's ratio as

$$
\nu^{[i, j]}=-\frac{\epsilon_{x x}^{[i, j]}}{\epsilon_{y y}^{[i, j]}},
$$

and of compaction as

$$
\psi^{[i, j]}=\frac{A_{0}^{[i, j]}-A^{[i, j]}}{A_{0}^{[i, j]}}=1-\left(1+\epsilon_{x x}^{[i, j]}\right)\left(1+\epsilon_{y y}^{[i, j]}\right),
$$

$A^{[i, j]}$ and $A_{0}^{[i, j]}$ denoting the current and initial area of the $[i-t h$, $j-t h]$ RVE. Then the ensemble averages $\bar{\epsilon}_{y y}=\left\langle\epsilon_{y y}^{[i, j]}\right\rangle, \bar{\nu}=<$ $\nu^{[i, j]}>, \bar{\psi}=<\psi^{[i, j]}>$ for the nine central RVEs under consideration were computed.

The evolution of the Poisson's ratio $\bar{\nu}$ as function of the local engineering strain $\bar{\epsilon}_{y y}$ is presented in Fig. 3(c). The difference between structure $\mathrm{C}$ and structures $\mathrm{A}$ and $\mathrm{B}$ is striking; while in structures $\mathrm{A}$ and $\mathrm{B}$ noticeable lateral contraction induced by buckling is observed, eventually leading to negative values of Poisson's ratio, the Poisson's ratio in structure $\mathrm{C}$ is only marginally affected by deformation and buckling. Moreover, it is interesting to observe that the lateral contraction in structure B is remarkably larger than that observed in structure A, leading to larger negative values of Poisson's ratio.

The evolution of the compaction $\bar{\psi}$ for the three structures (Fig. 3(d)) shows similar features to that of the Poisson's ratio. Microscopic instabilities in structure A and B are found to lead to a significant increase in compaction, revealing the important role played by buckling in the design of novel soft foldable systems. As clearly shown in Figs. 2 and 3, using mechanical instabilities and different pore shapes, materials and devices can be designed capable of dramatic area and shape change in response to an external stimulus, opening avenues for novel active and soft foldable structures.

Our results demonstrate the important role of the shape of the pores on the response of $2 \mathrm{D}$ porous structures. We reveal that the shape of the pores can be effectively used to tune their lateral contraction and compaction. To fully unravel the effect of the shape of the holes on the physical characteristics of the structures, we proceed by systematically investigating the effect of porosity $\phi$ on their response. Since our results clearly show that the finite element simulations were able to accurately reproduce the experimental results, we now investigate numerically the response of structures characterized by porosity $\phi$ ranging between 0.4 and 0.5 . Note that smaller values of porosity would facilitate macroscopic instability [17], leading to structures characterized by limited compaction. On the other hand, higher levels of porosity would lead to structures characterized by very thin ligaments, making them fragile. 

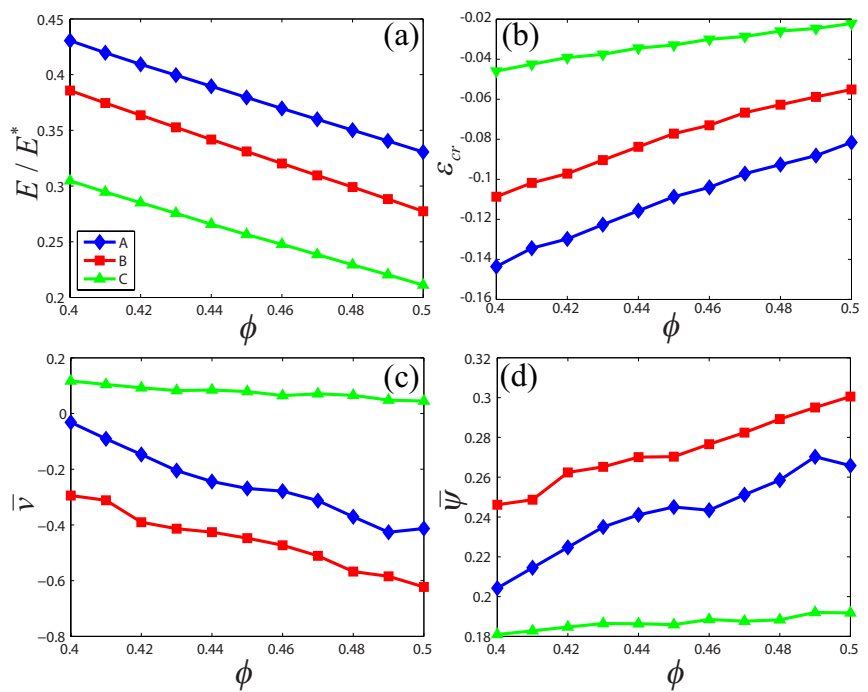

Fig. 4. Results of the numerical investigation on the effect of the porosity $\phi$ for the three structures. (a) Effective modulus $E / E^{*}$. (b) Critical strain $\epsilon_{c r}$. (c) Poisson's ratio $\bar{\nu}$ at a local strain $\bar{\epsilon}_{y y}=-0.2$. (d) Compaction $\bar{\psi}$ at a local strain $\bar{\epsilon}_{y y}=-0.2$.

In Fig. 4(a) the evolution of the initial elastic modulus $E$, normalized by the Young's modulus of the bulk material $E^{*}$, is reported as a function of the porosity $\phi$. The pore shape is found to strongly affect $E / E^{*}$; structures $\mathrm{B}$ and $\mathrm{C}$ both show an initial elastic modulus significantly lower than structure A and similarly we may expect to exploit the pore shape to design structures with stiffer initial elastic response.

Focusing on the non-linear response of the structures, Fig. 4(b) shows the buckling strain $\epsilon_{c r}$ for the three structures as a function of $\phi$. Over the explored range of porosity, we found that structures $\mathrm{A}$ and $\mathrm{B}$ are always characterized by microscopic modes, while for structure $\mathrm{C}$ a macroscopic mode is observed. This difference is reflected by the different dependence of $\epsilon_{c r}$ by $\phi$ : for structure $\mathrm{C} \epsilon_{c r}$ in only marginally affected by $\phi$, while it shows a much stronger dependency for structures $A$ and $B$.

Finally in Figs. 4(c) and (d) we investigated the effect of porosity on lateral contraction and compaction at a fixed local strain $\bar{\epsilon}_{y y}=-0.2$. Structure $\mathrm{C}$ is found to be characterized by a limited compaction over the entire range of porosity explored in this study. Differently, we observe a significant increase of $\bar{\nu}$ and $\bar{\psi}$ in structures A and B with increased values of porosity. Moreover, the results clearly show the superior response of structure B in terms of compaction and lateral contraction over the entire range of porosity. More precisely, at a given level of porosity the negative Poisson's ratio and compaction for structure B are found to be more than $20 \%$ larger than those of structure A. Thus the numerical results strongly confirm the important effect of pore shape both on the initial and non-linear response of the structures and demonstrate that shape can be exploited to fine tune their mechanical response.

We have shown that cellular solids, which comprise a solid matrix with a square array of holes, open avenues for the design of novel soft and foldable structures. Unlike many other examples of foldable structures, our system does not contain rigid links, but comprises continuous $2 \mathrm{D}$ soft and porous structures and takes advantage of mechanical instabilities, allowing the actuation to be fast, reversible and applicable over a wide range of length scales. Our results demon- strate that by simply changing the shape of the holes the response of porous structure can be easily tuned and soft structures with optimal compaction can be designed. Surprisingly, we show that circular holes do not lead to optimal response and that the compaction of the system can be significantly improved through a careful design of the pore shape. The insights gained by performing a numerical parametric exploration serve as an important design guideline in fabricating practical materials towards applications.

\section{Materials and Methods}

Materials. A silicone-based rubber (Elite Double 8, Zhermack) was used to cast the experimental specimen. The mechanical response of the material was characterized performing uniaxial tensile tests on samples with rectangular cross-section and shoulders. In addition, an extensometer was used to provide a more accurate measure of the stretch. The material response up to an applied stretch $\lambda=2$ was found to be well captured using a nearly incompressible NeoHookean model, whose strain energy is $W=\mu_{0} / 2\left(\bar{I}_{1}-3\right)+K_{0} / 2(J-1)^{2}$ , with $\mu_{0}=0.33 \mathrm{kPa}$ and $K_{0}=555 \mathrm{kPa}$. Here, $\bar{I}_{1}=\operatorname{tr}\left[\operatorname{dev}\left(\mathbf{F}^{T} \mathbf{F}\right)\right]$ $J=\operatorname{det} \mathbf{F}, \quad \mathbf{F}$ is the deformation gradient and $\mu_{0}$ and $K_{0}$ denote the shear modulus and bulk modulus at zero strain, respectively. Note that Young's modulus $E^{*}$ and Poisson ratio $\nu^{*}$ at zero strain can be easily obtained as $E^{*}=9 K_{0} \mu_{0} /\left(3 K_{0}+\mu_{0}\right)$ and $\nu^{*}=0.5\left(3 K_{0}-2 \mu_{0}\right) /\left(3 K_{0}+\mu_{0}\right)$.

Sample manufacture. Molds were fabricated using laser-cutting. Each mold comprised a base, four lateral walls and hundred spines to shape the contour of the pores. All parts were laser-cut from acrylic plates and assembled together. Spines with desired thickness were fabricated stacking several layers of acrylic plates and were inserted into the base using a hexagonal shaped key to orient them. Each mold comprised a 10×10 square array of holes with center-to-center spacing $L_{0}=10 \mathrm{~mm}$. The mold was open to the air, and the casted mixture was allowed to set at room temperature for 20 minutes. After demolding, the side walls were cut from the sample, leaving seven columns of seven holes, flanked by a column/row of seven semi holes on either sides (Fig. 2). The specimens for structures A, B and C measured $80 \times 80 \times 14.5 \mathrm{~mm}, 80 \times 80 \times 18.5 \mathrm{~mm}$, $80 \times 80 \times 15.5 \mathrm{~mm}$, respectively. The porosity of three specimens was measured as $\phi^{A}=0.46, \phi^{B}=0.47$ and $\phi^{C}=0.44$.

Testing and analysis. Compression tests were performed using an Instron machine with a $10 \mathrm{~N}$ load cell. During the tests the specimens were positioned on an horizontal plate and compressed by a flat fixture mounted to the vertical compressing head. Both fixtures were made of transparent acrylic. Note that the specimens were not clamped to the two horizontal fixtures and that friction between the sample and fixture surface was enough to fix the position of the specimen top and bottom boundary (no lubrificant was used on the horizontal surfaces). The parallelism of the two horizontal flat fixtures was carefully monitored during the tests. The specimen during the test was held by an acrylic plate at the back directly mounted to the load cell to avoid out-of-plane buckling. The sample faces were covered with vaseline to reduce any frictional effects resulting during the loading process. The compression tests were performed at $20 \mathrm{~mm} / \mathrm{min}$ until the maximum load of the load cell was reached. The results were independent of the rate of change of displacement and hence a good approximation to the rate independent conditions of the numerical investigation was obtained in the experiments. The load associated with the displacement was recorded and used to produce stress-strain curves for the compression process. During the test, a Nikon D90 SLR camera facing the specimen was used to take pictures and videos. Marking the corners of RVEs in our specimen with black dots enabled us to evaluate the geometric change quantitatively with a post-processing code in MATLAB.

\section{ACKNOWLEDGMENTS.}

This work has been supported by the Harvard Materials Research Science and Engineering Center under NSF award number DMR-0820484 and by the Kavli Institute at Harvard University. The authors are grateful to discussions with Pedro M. Reis (M.I.T.) 
1. S. Pellegrino (Ed.)(2001) Deployable structures CISM International Centre for Mechanical Sciences 412.

2. J. Clinton, NASA Report CR-1735, 1971

3. S. Agrawal, S. Kumar, S. Yim, Journal of Mechanical Design 2002, 124, 473478.

4. C. Hoberman, USA patent $1990,4,942,700$.

5. F. Kovacs, T. Tarnai, P. Fowler, S. Guest, Int. J. Solids. Struct. 2004, 41, 11191137.

6. K. Wohlhart, Proceedings of the Second Workshop on Computational Kinematics 2001, 239248.

7. M. Weintraub New Phytologist 1952, 50, 357-382.

8. H.S. Patil , V. Siddharth Journal of Bionic Engineering 2007, 4, 19-23.

9. K. Bertoldi, M.C. Boyce, Physical Review B 2008, 78, 184107.

10. Y. Forterre, J.M. Skotheim , J. Dumais , L. Mahadevan, Nature 2005, 433, 421-425.

11. R.L. Sutherland, L.M. Mathger, R.T. Hanlon, A.M. Urbas, M.O. Stone, J. Opt. Soc. Am. 2008, 25, 2044-2054.

12. J.H. Jang, C.K. Ullal, T. Gorishnyy, V.V. Tsukruk, E.L. Thomas, Nano Letters 2006, 6 , 4,740-743.

13. S. Singamaneni, V.V. Tsukruk, Soft Matter 2010, 6, 5681-5692

14. T. Mullin, S. Deschanel, K. Bertoldi, M.C. Boyce, Physical Review Letters 2007, 99, 084301.
15. Y. Zhang, E.A. Matsumoto, A. Peter, P.C. Lin, R.D. Kamien, S. Yang, Nano Letters 2008, 8, 1192-1196.

16. S. Singamaneni, K. Bertoldi, S. Chang, J.H. Jang, E.L. Thomas, M.C. Boyce, V.V. Tsukruk. ACS Appl. Mater. Interfaces 2009, 1, 1, 42-47.

17. K. Bertoldi, P.M. Reis, S. Willshaw, T. Mullin, Advanced Materials 2010, 22, 361-366

18. J.H. Jang, C.Y. Koh, K. Bertoldi, M.C. Boyce, E.L. Thomas, Nano Letters 2009, 9, 2113-2119.

9. D. Krishnan, H. T. Johnson, Journal of the Mechanics and Physics of Solids 2009, 57, 15001513.

20. H.A. Eschenauer, N. Olhoff, Appl. Mech. Rev. 2001, 54, 331-391.

21. M.P. Bendsoe, O. Sigmund, Topology Optimization, Theory, Methods and Applications. Springer 2003

22. R.T. Haftka, R.V. Grandhi Computer Methods in Applied Mechanics and Engineering 1985, 57, 91-106.

23. D.C. Lagoudas (Ed.), Shape Memory Alloys: Modeling and Engineering Applications. Springer 2008.

24. L.J. Gibson, M.F. Ashby, Cellular Solids: Structure and Properties. Cambridge University Press 1997. 